\title{
Correction to: Flag-transitive block designs and unitary groups
}

\author{
Seyed Hassan Alavi ${ }^{1}$. Mohsen Bayat ${ }^{1}$. Ashraf Daneshkhah ${ }^{1}$
}

Published online: 12 April 2021

๑) Springer-Verlag GmbH Austria, part of Springer Nature 2021

\section{Correction to: Monatsh Math https://doi.org/10.1007/s00605-020-01421-8}

\begin{abstract}
In this note, we cover a gap in the proof of [2, Proposition 4.3]. In conclusion, Theorem 1.1 in [2] is revisited: if $\mathcal{D}$ is a 2-design with $\operatorname{gcd}(r, \lambda)=1$ and $G$ is a flag-transitive almost simple automorphism group of $\mathcal{D}$ whose socle is $\operatorname{PSU}(n, q)$ with $(n, q) \neq$ $(3,2)$, then $\mathcal{D}$ belongs to one of the three infinite families of Hermitian unitals, WittBose-Shrikhande spaces and 2-designs with parameters $\left(q^{3}+1, q, q-1\right)$, or it is isomorphic to a design with parameters $(6,3,2),(7,3,1),(8,4,3),(10,6,5),(11,5$, $2)$ or $(28,7,2)$.
\end{abstract}

Keywords 2-Design, Flag-transitive, Automorphism group, Almost simple group, Unitary group

Mathematics Subject Classification 05B05 · 05E18· 20D05

We would like to thank the editors for their helpful comments. We would also like to thank Shenglin Zhou for pointing out this missing part while we were working on a joint project.

The original article can be found online at https://doi.org/10.1007/s00605-020-01421-8.

Seyed Hassan Alavi

alavi.s.hassan@basu.ac.ir; alavi.s.hassan@gmail.com

Mohsen Bayat

m.bayat@sci.basu.ac.ir

Ashraf Daneshkhah

adanesh@basu.ac.ir

1 Department of Mathematics, Faculty of Science, Bu-Ali Sina University, Hamedan, Iran 


\section{Introduction}

A 2-design $\mathcal{D}$ with parameters $(v, k, \lambda)$ is a pair $(\mathcal{P}, \mathcal{B})$ with a set $\mathcal{P}$ of $v$ points and a set $\mathcal{B}$ of $b$ blocks such that each block is a $k$-subset of $\mathcal{P}$ and each two distinct points are contained in $\lambda$ blocks. We say $\mathcal{D}$ is nontrivial if $2<k<v-1$, and symmetric if $v=b$. Each point of $\mathcal{D}$ is contained in exactly $r$ blocks which is called the replication number of $\mathcal{D}$. A flag of $\mathcal{D}$ is a point-block pair $(\alpha, B)$ such that $\alpha \in B$. An automorphism of a 2-design $\mathcal{D}$ is a permutation of the points permuting the blocks and preserving the incidence relation. The full automorphism group $\operatorname{Aut}(\mathcal{D})$ of $\mathcal{D}$ is the group consisting of all automorphisms of $\mathcal{D}$. For $G \leq \operatorname{Aut}(\mathcal{D}), G$ is called flag-transitive if $G$ acts transitively on the set of flags and $G$ is said to be point-primitive if it is primitive on $\mathcal{P}$. In this note, we cover a gap in the proof of [2, Proposition 4.3]. Therefore, we correct Theorem 1.1 in [2] as below:

Theorem 1.1 Let $\mathcal{D}$ be a nontrivial 2-design with $\operatorname{gcd}(r, \lambda)=1$, and let $\alpha$ be a point of D. Suppose that $G$ is an automorphism group of $\mathcal{D}$ whose socle is $X=\operatorname{PSU}(n, q)$ with $(n, q) \neq(3,2)$. If $G$ is flag-transitive, then $\lambda \in\{1,2,3,5\}$ and $v, k, \lambda, X_{\alpha}$ and $X$ are as in one of the lines in Table 1 or one of the following holds:

(a) $\mathcal{D}$ is a Witt-Bose-Shrikhande space with parameters $\left(2^{n-1}\left(2^{n}-1\right), 2^{n-1}, 1\right)$ and $X$ is $\operatorname{PSU}\left(2,2^{n}\right)$ with $n \geq 3$;

(b) $\mathcal{D}$ is a Hermitian unital $\mathcal{U}_{H}(q)$ with parameters $\left(q^{3}+1, q+1,1\right)$ and $X$ is $\operatorname{PSU}(3, q)$;

(c) $\mathcal{D}$ is a 2-design with parameters $\left(q^{3}+1, q, q-1\right)$ and $X$ is $\operatorname{PSU}(3, q)$, and the point set of $\mathcal{D}$ is the point set of a Hermitian unital $\mathcal{U}_{H}(q)$ and the block set is $(\ell \backslash\{\gamma\})^{G}$ where $\ell$ is a line of $\mathcal{U}_{H}(q)$ and $\gamma \in \ell$.

Remark 1.1 We remark here that the class $\mathcal{C}_{5}$ should be excluded from [2, Lemma 3.11] when $H$ is of type $\mathrm{GU}_{n}\left(q_{0}\right)$ with $q=q_{0}^{t}$ and $t$ odd prime. However, this change does not affect the proof of [2, Proposition 4.3] as the large subgroup condition in [2, Lemma 3.6] implies in this case that $t=3$ which was handled in [2, Propositions 4.1 and 4.3].

It is worth noting by [6] that there is a general construction method for 2-designs from linear space: For a $2-(v, k, 1)$ design $\mathcal{S}=(\mathcal{P}, \mathcal{L})$ with $k \geq 3$, let $\mathcal{B}=\{\ell \backslash\{\alpha\} \mid$ $\ell \in \mathcal{L}, \alpha \in \ell\}$ and $\mathcal{D}(\mathcal{S})=(\mathcal{P}, \mathcal{B})$. Then [6, Proposition 4.1] implies that $\mathcal{D}(\mathcal{S})$ is a $2-(v, k-1, k-2)$ design, and moreover, that $G$ is flag-transitive on $\mathcal{D}(\mathcal{S})$ whenever $G \leq \operatorname{Aut}(\mathcal{S})$ is flag-transitive on $\mathcal{S}$ and induces a 2-transitive action on each line of $\mathcal{S}$. Therefore, the design in Theorem 1.1 can be obtained in this way by taking $\mathcal{S}$ as the Hermitian unital $\mathcal{U}_{H}(q)$.

\section{Proof of Theorem 1.1}

In this section, we prove Proposition 2.1 below, and this together with [2, Proposition 4.2] will prove Theorem 1.1. In order to prove Proposition 2.1, we first need to introduce the Hermitian unitals. Here, we follow the same terminology as in [8] with a few exceptions in our notation. 
Table 1 Some nontrivial 2-design with $\operatorname{gcd}(r, \lambda)=1$

\begin{tabular}{llllllllll}
\hline Line & $v$ & $b$ & $r$ & $k$ & $\lambda$ & $X_{\alpha}$ & $X$ & Designs & References \\
\hline 1 & 6 & 10 & 5 & 3 & 2 & $\mathrm{D}_{10}$ & $\operatorname{PSU}(2,5)$ & - & {$[4,12]$} \\
2 & 7 & 7 & 3 & 3 & 1 & $\operatorname{Sym}_{4}$ & $\operatorname{PSU}(2,7)$ & $\operatorname{PG}(2,2)$ & {$[1,4,9]$} \\
3 & 8 & 14 & 7 & 4 & 3 & $\mathrm{C}_{7}: \mathrm{C}_{3}$ & $\operatorname{PSU}(2,7)$ & - & \\
4 & 10 & 15 & 9 & 6 & 5 & $\mathrm{C}_{3}^{2}: \mathrm{C}_{4}$ & $\operatorname{PSU}(2,9)$ & - & {$[1,4,12]$} \\
5 & 11 & 11 & 5 & 5 & 2 & $\mathrm{Alt}_{5}$ & $\operatorname{PSU}(2,11)$ & Hadamard & {$[1,4,9]$} \\
6 & 28 & 36 & 9 & 7 & 2 & $\mathrm{D}_{18}$ & $\operatorname{PSU}(2,8)$ & - & {$[4,12]$} \\
\hline
\end{tabular}

Let $q=p^{a}>2$ with $p$ a prime. The mapping $x \mapsto x^{q}$ is an automorphism of the Galois field $\mathbb{F}_{q^{2}}$, which we will write as $x^{q}=\bar{x}$ occasionally. The Galois field $\mathbb{F}_{q}$ is then the fixed field of this automorphism. Let $V$ be a three-dimensional vector space over $\mathbb{F}_{q^{2}}$ and $\varphi$ a nondegenerate $\sigma$-Hermitian form on $V$. The full unitary group $\Gamma \mathrm{U}(3, q)$ consists of those semilinear transformations of $V$ that induce a collineation of $\operatorname{PG}\left(2, q^{2}\right)$ which commutes with $\varphi$. The general unitary group $\mathrm{GU}(3, q)=\Gamma \mathrm{U}(3, q) \cap \mathrm{GL}\left(3, q^{2}\right)$ is the group of nonsingular linear transformations of $V$ leaving $\varphi$ invariant. The projective unitary group $\operatorname{PGU}(3, q)$ is the quotient group $\mathrm{GU}(3, q) / Z$, where $Z=\left\{a I \mid a \in \mathbb{F}_{q^{2}}, a^{q+1}=1\right\}$ is the center of $\mathrm{GU}(3, q)$ and $I$ the identity transformation. The special projective unitary group $\operatorname{PSU}(3, q)$ is the quotient group $\mathrm{SU}(3, q) /(Z \cap \mathrm{SU}(3, q))$, where $\mathrm{SU}(3, q)$ is the subgroup of $\mathrm{GU}(3, q)$ consisting of linear transformations of unit determinant. The group $\operatorname{PSU}(3, q)$ is equal to $\operatorname{PGU}(3, q)$ if 3 is not a divisor of $q+1$, and is a subgroup of $\operatorname{PGU}(3, q)$ of index 3 otherwise. It is well-known that the automorphism group of $\operatorname{PSU}(3, q)$ is equal to $\operatorname{P\Gamma U}(3, q):=\operatorname{PGU}(3, q)\left\langle\sigma_{p}\right\rangle$, where $\sigma_{p}: x \mapsto x^{p}$ is the Frobenius map. By [8, Lemma 4.1], we choose an appropriate basis $\left\{e_{1}, e_{2}, e_{3}\right\}$ for $V$ with corresponding Hermitian matrix of $\varphi$ by

$$
\left(\begin{array}{lll}
0 & 0 & 1 \\
0 & 1 & 0 \\
1 & 0 & 0
\end{array}\right) .
$$

If $u=\left(x_{1}, x_{2}, x_{3}\right)$ and $v=\left(y_{1}, y_{2}, y_{3}\right)$ are vectors in $V$, then $\varphi(u, v)=x_{1} y_{3}^{q}+$ $x_{2} y_{2}^{q}+x_{3} y_{1}^{q}$. A vector $u \in V$ is called isotropic if $\varphi(u, u)=0$ and nonisotropic otherwise. Let

$$
\mathcal{P}=\left\{\langle 0,0,1\rangle,\langle 1, a, b\rangle \mid a, b \in \mathbb{F}_{q^{2}} \text { and } a^{q+1}+b+b^{q}=0\right\},
$$

where $\langle a, b, c\rangle$ denotes the 1-dimensional subspace of $V$ spanned by $(a, b, c) \in V$. The elements of $\mathcal{P}$ are called the absolute points. It is well-known that $|\mathcal{P}|=q^{3}+1$, $\operatorname{PSU}(3, q)$ is 2 -transitive on $\mathcal{P}$, and $\mathrm{P} \Gamma \mathrm{U}(3, q)$ leaves $\mathcal{P}$ invariant. Denote

$$
\infty:=\langle 0,0,1\rangle \text { and } 0:=\langle 1,0,0\rangle,
$$


and set

$$
\Delta:=\left\{\langle 1,0, b\rangle \mid b \in \mathbb{F}_{q^{2}} \text { and } b+b^{q}=0\right\},
$$

and let $H$ be the point-stabiliser of $\infty$ in $X=\operatorname{PSU}(3, q)$, that is to say, $H=X_{\infty}$. By [8], we have the following information about these groups and their actions on $\mathcal{P}$ :

(a) $H=Q L$, where $Q$ is a normal subgroup of $H$ of order $q^{3}$ which acts regularly on $\mathcal{P} \backslash\{\infty\}$ and $L=X_{\infty, 0}$ which is a cyclic subgroup of $H$ of order $\left(q^{2}-\right.$ $1) / \operatorname{gcd}(3, q+1)$

(b) $L=X_{\infty, 0}$ has two trivial orbits $\{0\},\{\infty\}$, one nontrivial orbit $\Delta \backslash\{0\}=\{\langle 1,0, b\rangle \mid$ $0 \neq b \in \mathbb{F}_{q^{2}}$ and $\left.b+b^{q}=0\right\}$ of length $q-1$, and its remaining nontrivial orbits are of length $\left(q^{2}-1\right) / \operatorname{gcd}(3, q+1)$;

(c) $P=Z(Q)=[Q, Q]$ is a subgroup of $Q$ of order $q$ fixing $\infty$ and acting transitively on $\Delta$ defined in (2);

(d) $P L=X_{\infty, \ell(\infty)}$ is transitive on $\Delta$ and it is of order $q\left(q^{2}-1\right) / \operatorname{gcd}(3, q+1)$, where $\ell(\infty)=\{\infty\} \cup \Delta$, that is to say,

$$
\ell(\infty)=\{\infty\} \cup\left\{\langle 1,0, b\rangle \mid b \in \mathbb{F}_{q^{2}}, b+b^{q}=0\right\} ;
$$

The Hermitian unital $\mathcal{U}_{H}(q)$ is defined to be the block design with the point set $\mathcal{P}$ in which a subset of $\mathcal{P}$ is a block (called a line) precisely when it is the set of absolute points contained in some $\langle u, v\rangle$. We know by [7,8,11] that $\mathcal{U}_{H}(q)$ is a linear space with $q^{3}+1$ points, $q^{2}\left(q^{2}-q+1\right)$ lines, $q+1$ points in each line, and $q^{2}$ lines on each point. It was proved in $[8,11]$ that $\operatorname{Aut}\left(\mathcal{U}_{H}(q)\right)=\operatorname{P\Gamma U}(3, q)$. Thus, every $G$ with $X=\operatorname{PSU}(3, q) \leq G \leq \operatorname{P\Gamma U}(3, q)$ acts 2 -transitively on the point set of $\mathcal{U}_{H}(q)$. This implies that $G$ is also block-transitive and flag-transitive on $\mathcal{U}_{H}(q)$. A line of $\mathrm{PG}\left(2, q^{2}\right)$ contains either one absolute point or $q+1$ absolute points. In the latter case, the set of such $q+1$ absolute points is a line of $\mathcal{U}_{H}(q)$, and all lines of $\mathcal{U}_{H}(q)$ are of this form. In particular, $\ell(\infty)$ defined in (3) is a line of $\mathcal{U}_{H}(q)$ containing $\infty$ (see [8, Lemma 2.5]). Moreover, the line stabiliser $X_{\ell(\infty)}$ is transitive on $\ell(\infty)$ and $P \leq X_{\infty, \ell(\infty)}$ is transitive on $\ell(\infty) \backslash\{\infty\}$, and hence $X_{\ell(\infty)}$ is 2-transitive on $\ell(\infty)$. Since $X$ is flag-transitive, for each line $\ell$ of $\mathcal{U}_{H}(q)$, we conclude that $X_{\ell}$ is 2-transitive on $\ell$.

Suppose now that $B=\ell(\infty) \backslash\{0\}$. The information given above are useful to observe that $X_{\infty, B}=X_{\infty, 0}$ and $X_{B} \leq X_{\ell(\infty)}$, and so $X_{B}=X_{0, B}$ is a subgroup of index $q+1$ in $X_{\ell(\infty)}$ and $\left|X_{B}: X_{\infty, B}\right|=q$. Note that $X$ is 2-transitive on $\mathcal{P}$. If $\mathcal{B}=B^{X}$, then $(\mathcal{P}, \mathcal{B})$ is a 2 -design with parameters $\left(q^{3}+1, q, q-1\right)$, and hence this gives an explicit construction for the design that appears in Theorem 1.1(c).

We are now ready to revisit Proposition 4.3 in [2], and prove Proposition 2.1 below. In what follows, we frequently use the results mentioned above about the Hermitian unitals and their automorphism groups.

Proposition 2.1 Let $\mathcal{D}$ be a nontrivial 2-design with $\operatorname{gcd}(r, \lambda)=1$. Suppose that $G$ is an automorphism group of $\mathcal{D}$ whose socle is $X=\operatorname{PSU}(n, q)$ with $n \geq 3$ and $(n, q) \neq(3,2)$. If $G$ is flag-transitive, then $X$ is $\operatorname{PSU}(3, q)$, and one of the following holds: 
(a) $\mathcal{D}$ is a Hermitian unital with parameters $\left(q^{3}+1, q+1,1\right)$;

(b) $\mathcal{D}$ is a 2-design with parameters $\left(q^{3}+1, q, q-1\right)$, and the point set of $\mathcal{D}$ is the point set of a Hermitian unital $\mathcal{U}_{H}(q)$ and the block set is $(\ell \backslash\{\gamma\})^{G}$ where $\ell$ is a line of $\mathcal{U}_{H}(q)$ and $\gamma \in \ell$.

Proof Suppose that $H=G_{\alpha}$ with $\alpha$ a point of $\mathcal{D}$. If $H$ is not a parabolic subgroup $P_{m}$, then we follow the same argument as in [2, Proposition 4.3] which leads to no possible parameters. Therefore, considering Remark 1.1, we only need to deal with the case where $H$ is isomorphic to $P_{m}$, for some $2 m \leq n$. In this case, by the same argument as in [2, Proposition 4.3], the inequality $v<r^{2}$ restricts to the case where $n=3$, that is to say, $X=\operatorname{PSU}(3, q)$ and $H \cap X \cong \hat{q} q^{3}\left(q^{2}-1\right)$ in which case $v=q^{3}+1$. If $\lambda=1$, then by [10], $\mathcal{D}$ is a Hermitian unital as in part (a). Suppose now that $\lambda>1$. Here, $X$ acts 2 -transitively on the point set of $\mathcal{D}$, and this action is permutationally isomorphic to the action of $X$ on the set $\mathcal{P}$ as in (1). Therefore, without loss of generality, we can identify the point set of $\mathcal{D}$ with $\mathcal{P}$, and take $\alpha:=\infty$. Since $\operatorname{gcd}(r, \lambda)=1,[5,1.2 .8]$ implies that $X$ is flag-transitive, and hence we can also assume that $G=X$, and so $H=X_{\infty} \cong \hat{q^{3}}\left(q^{2}-1\right)$. Let $B$ be a block containing $\infty$, and let $\ell:=\ell(\infty)$ be a line in $\mathcal{U}_{H}(q)$ passing through $\infty$. Since $r$ divides $v-1=q^{3}$ where $q=p^{3 a}$, it follows that $r=p^{t}$, for some $t \leq 3 a$. Since also $b=r v / k$, we have that $\left|X_{B}\right|=|X| / b=k p^{3 a-t}\left(q^{2}-1\right)$. By inspecting the maximal subgroups of $X$ from [3, Table 8.5], we then conclude that $X_{B}$ is contained in $X_{\ell}$ which is isomorphic to $\mathrm{GU}_{2}(q)$. Since $X_{B}$ is contained in a maximal subgroup $M$ of $X_{\ell}$ and $X_{\ell}$ is 2-transitive on $\ell, M$ is a point-stabiliser of $X_{\ell}$. By possibly replacing $B$ with its conjugate, we can assume that $X_{B} \leq X_{0, \ell}$. Thus $X_{\infty, B}$ is contained in $X_{\infty, 0, \ell}=X_{\infty, 0}$. Since $b k=v r=p^{t}\left(q^{3}+1\right)$ and $b k=\left|X: X_{B}\right| \cdot\left|X_{B}: X_{\infty, B}\right|=\left|X: X_{\infty, B}\right|$, we conclude that $\left|X_{\infty, B}\right|=p^{3 a-t}\left(q^{2}-1\right) / d$. Recall that $X_{\infty, B} \leq X_{\infty, 0}$ and $X_{\infty, 0}$ is a cyclic group of order $\left(q^{2}-1\right) / d$. Therefore, $X_{\infty, B}=X_{\infty, 0}$. We know that $\left|X_{0, \ell}: X_{\infty, 0}\right|=q$. Since $X_{0, B}$ is contained in $X_{0, \ell}$, it follows that $k=\left|X_{B}: X_{\infty, B}\right|=\left|X_{B}: X_{\infty, 0}\right| \leq$ $\left|X_{0, \ell}: X_{\infty, 0}\right|=q$, that is to say, $k \leq q$. Recall that $X_{\infty, B}=X_{\infty, 0}$. Then $X_{\infty, 0}$ fixes $B$, and so $B \backslash\{\infty\}$ is a union of nontrivial $X_{\infty, 0}$-orbits. We know that $X_{\infty, 0}$ fixes $\infty$ and 0 , and it has one nontrivial orbit of length $q-1$ and its remaining nontrivial orbits are of length $\left(q^{2}-1\right) / d$. Since $k \leq q$, we conclude that $B \backslash\{\infty\}$ is the nontrivial $X_{\infty, 0^{-}}$ orbit $\ell \backslash\{\infty, 0\}$ of length $q-1$. Therefore, $B=\ell \backslash\{0\}$. Indeed, $B=\{\infty\} \cup(\Delta \backslash\{0\})$, where $\Delta$ is as in (2). This implies that $k=q, b=q^{2}\left(q^{3}+1\right)$ and $\lambda=q-1$. In conclusion, $\mathcal{D}$ is a 2-design with parameters $\left(q^{3}+1, q, q-1\right)$. If $X$ fixes 0 and $\ell$, then it fixes $\ell \backslash\{0\}$. Thus $X_{0, \ell} \leq X_{B}$, and since $X_{0, \ell}$ is transitive on $B=\ell \backslash\{0\}$, it follows that $X_{B}$ is transitive on $B=\ell \backslash\{0\}$, and hence $X$ is flag-transitive. Therefore, $\mathcal{D}$ is a 2-design with parameters $\left(q^{3}+1, q, q-1\right)$ whose points are the points of $\mathcal{U}_{H}(q)$ and $\mathcal{B}=B^{X}$, where $B=\ell \backslash\{0\}$ with $\ell$ a line of $\mathcal{U}_{H}(q)$.

\section{References}

1. Alavi, S.H., Bayat, M., Daneshkhah, A.: Symmetric designs admitting flag-transitive and pointprimitive automorphism groups associated to two dimensional projective special groups. Des. Codes Cryptogr. 79(2), 337-351 (2016). https://doi.org/10.1007/s10623-015-0055-9 
2. Alavi, S.H., Bayat, M., Daneshkhah, A.: Flag-transitive block designs and unitary groups. Monatshefte für Mathematik 193(3), 535-553 (2020). https://doi.org/10.1007/s00605-020-01421-8

3. Bray, J.N., Holt, D.F., Roney-Dougal, C.M.: The maximal subgroups of the low-dimensional finite classical groups, London Mathematical Society Lecture Note Series, vol. 407. Cambridge University Press, Cambridge (2013). https://doi.org/10.1017/CBO9781139192576.With a foreword by Martin Liebeck

4. Colbourn, C.J., Dinitz, J.H. (eds.): Handbook of combinatorial designs, second edn. Discrete Mathematics and its Applications (Boca Raton). Chapman \& Hall/CRC, Boca Raton, FL (2007)

5. Dembowski, P.: Finite geometries. Ergebnisse der Mathematik und ihrer Grenzgebiete, Band 44. Springer, Berlin (1968)

6. Devillers, A., Liang, H., Praeger, C.E., Xia, B.: On flag-transitive 2-( $v, k$, 2) designs. J. Combin. Theory Ser. A 177(105309), 45 (2021). https://doi.org/10.1016/j.jcta.2020.105309

7. Kantor, W.M.: Homogeneous designs and geometric lattices. J. Combin. Theory Ser. A 38(1), 66-74 (1985). https://doi.org/10.1016/0097-3165(85)90022-6

8. O’Nan, M.E.: Automorphisms of unitary block designs. J. Algebra 20, 495-511 (1972). https://doi. org/10.1016/0021-8693(72)90070-1

9. O’Reilly-Regueiro, E.: On primitivity and reduction for flag-transitive symmetric designs. J. Combin. Theory Ser. A 109(1), 135-148 (2005). https://doi.org/10.1016/j.jcta.2004.08.002

10. Saxl, J.: On finite linear spaces with almost simple flag-transitive automorphism groups. J. Combin. Theory Ser. A 100(2), 322-348 (2002). https://doi.org/10.1006/jcta.2002.3305

11. Taylor, D.E.: Unitary block designs. J. Comb. Theory Ser. A 16, 51-56 (1974). https://doi.org/10.1016/ 0097-3165(74)90071-5

12. Zhan, X., Zhou, S.: Non-symmetric 2-designs admitting a two-dimensional projective linear group. Des. Codes Cryptogr. 86(12), 2765-2773 (2018). https://doi.org/10.1007/s10623-018-0474-5

Publisher's Note Springer Nature remains neutral with regard to jurisdictional claims in published maps and institutional affiliations. 